\title{
Management of a congenital tracheoesophageal fistula in a young Spanish water dog
}

\author{
Pia S Kaminen ${ }^{1 *}$, Sanna J Viitanen ${ }^{1}$, Anu K Lappalainen ${ }^{1}$, Anja Kipar ${ }^{2}$, Minna M Rajamäki ${ }^{1}$ \\ and Outi M Laitinen-Vapaavuori ${ }^{1}$
}

\begin{abstract}
Background: Tracheoesophageal fistula (TEF) in dogs is a rare disease with only few reports in the literature. This report aims to contribute to the current literature on suitable diagnostic methods for TEF and to provide follow-up information after successful surgical treatment.

Case presentation: A seven-month-old intact female Spanish Water Dog was presented for further investigation of recurrent respiratory symptom. Bronchoscopy revealed a small hole-like defect in the tracheal wall at the bifurcation. The finding of the contrast material swallow study under fluoroscopy was indicative of a TEF. To further evaluate the connection between the trachea and esophagus, a computed tomography scan was performed. The TEF was surgically approached by thoracotomy through the right lateral sixth intercostal space. The fistula was identified, double ligated and divided. Histopathology confirmed the process to originate from the esophagus and to be patent. The dog was re-examined two weeks and ten months after surgery, with no evidence of recurring clinical signs.

Conclusions: Contrast material swallow study using fluoroscopy was the most reliable diagnostic method. Bronchoscopy may allow the fistula to be visualized, but due to a small fistular opening it can lead to a false negative result. Surgical correction by ligation and dividing of the fistula suggests a good prognosis for early diagnosed and operated TEF.
\end{abstract}

Keywords: Congenital tracheoesophageal fistula, Dog, Bronchoscopy, Fluoroscopy, Computed tomography, Histopathology

\section{Background}

An esophageal fistula is an abnormal communication between the esophagus and trachea, bronchus, lung parenchyma or, rarely, the skin [1]. Congenital and acquired tracheoesophageal or bronchoesophageal fistulas in dogs are rarely identified as predisposing factors for recurrent pneumonia [2-6]. To our knowledge, there have been only two previous reports describing tracheoesophageal fistula (TEF) in dogs, one congenital [2] and the other acquired due to an esophageal foreign body [5]. Due to the rarity of the entity, there is very little experience concerning diagnostic methods and surgical correction techniques for TEF in dogs. Therefore, this report aims to contribute to the current literature on suitable diagnostic methods for

\footnotetext{
* Correspondence: pia.kaminen@helsinki.fi

${ }^{1}$ Department of Equine and Small Animal Medicine, Faculty of Veterinary Medicine, Helsinki University, Helsinki, Finland

Full list of author information is available at the end of the article
}

TEF and to provide follow-up information after successful surgical treatment.

\section{Case presentation}

A seven-month-old, $13.2 \mathrm{~kg}$ intact female Spanish Water Dog was presented for further investigation of recurrent respiratory symptoms. Three weeks before referral, the dog had developed an acute onset of lethargy and coughing. Pneumonia was suspected, since chest radiographs showed consolidation of the lungs. Treatment with amoxicillin-clavulanic acid ${ }^{a}$ for ten days resulted in transient improvement in the clinical signs.

On presentation, lethargy, spontaneous cough, tachypnea (respiratory rate $60 / \mathrm{min}$ ), and moderate crackles on lung auscultation were noted. Coughing was provoked especially after drinking and tracheal palpation. The partial oxygen pressure in the arterial blood $\left(\mathrm{PaO}_{2}\right)$ was $80.5 \mathrm{mmHg}$. The C-reactive protein (CRP) level was markedly elevated 
(138 mg/l). Thoracic radiographs revealed alveolar consolidations in the left cranial and left caudal lung lobe, consistent with bronchopneumonia. In abdominal radiographs, the small intestine was gas filled. Supportive treatment and intravenous amoxicillin-clavulanic acid ${ }^{\mathrm{b}}$ administration were initiated.

The dog was anesthetized and bronchoscopy performed, using a 4.9-mm flexible bronchoscope ${ }^{\mathrm{c}}$. At the bifurcation, a small hole-like defect was detected in the tracheal wall (Figure 1). This defect was not visible after examination of the main bronchi (Figure 2). A moderate amount of yellow, viscous, foamy secretion was detected in the bronchial lumen of the left cranial lung lobe. Bronchoalveolar lavage (BAL) was performed on the left cranial lung lobe with a $1-\mathrm{ml} / \mathrm{kg}$ bolus of sterile warm $\left(37^{\circ} \mathrm{C}\right)$ saline $\mathrm{d}^{\mathrm{d}}$. The cytological examination revealed an elevated total nucleated cell count $(1750$ cells $/ \mu \mathrm{l})$ and a significantly elevated neutrophil proportion (64.7\%). Aerobic and anaerobic cultures were negative, but after enrichment, Pasteurella stomatis was isolated in pure culture. The mycoplasma culture isolated Mycoplasma spp.

To further investigate the defect in the tracheal wall, an upper gastrointestinal contrast material swallow (CS) study was performed with a 10-ml bolus of per orally administered barium contrast medium ${ }^{\mathrm{e}}$. A laterolateral thoracic radiograph obtained immediately after the barium swallow showed no leakage of the contrast medium outside the esophagus. Investigations were further continued by a CS study under fluoroscopy with the dog in a standing position. There was no evidence that undiluted

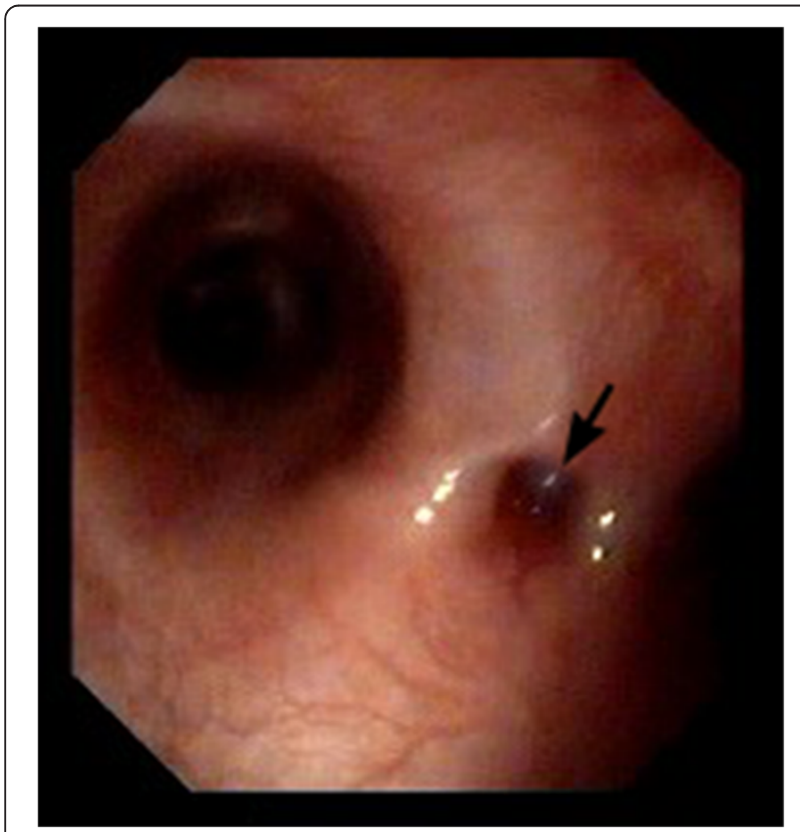

Figure 1 Endoscopic view of the tracheal bifurcation. A small hole-like tracheal wall defect is indicated with an arrow.

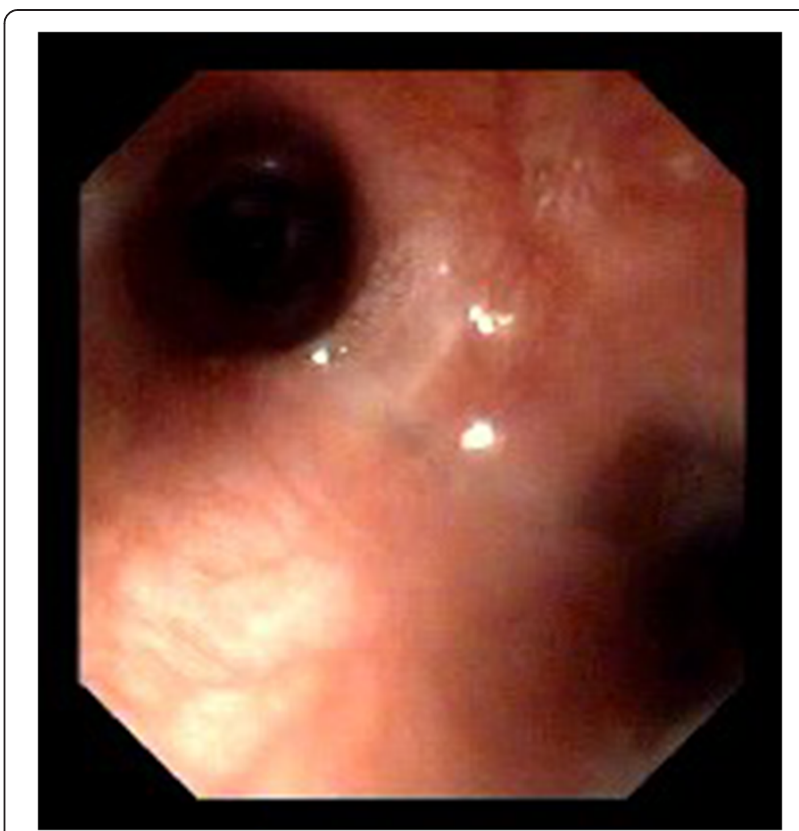

Figure 2 Endoscopic view of the tracheal bifurcation. The tracheal wall defect appeared closed after examination of the major bronchi, and as such could easily have been left unrecognized.

barium contrast medium leaked from the esophagus. However, when diluted 1:1 with water, a small amount of contrast medium was seen to escape ventrally from the esophagus, through a duct-like structure (Figure 3). During the procedure, the otherwise normal anatomic

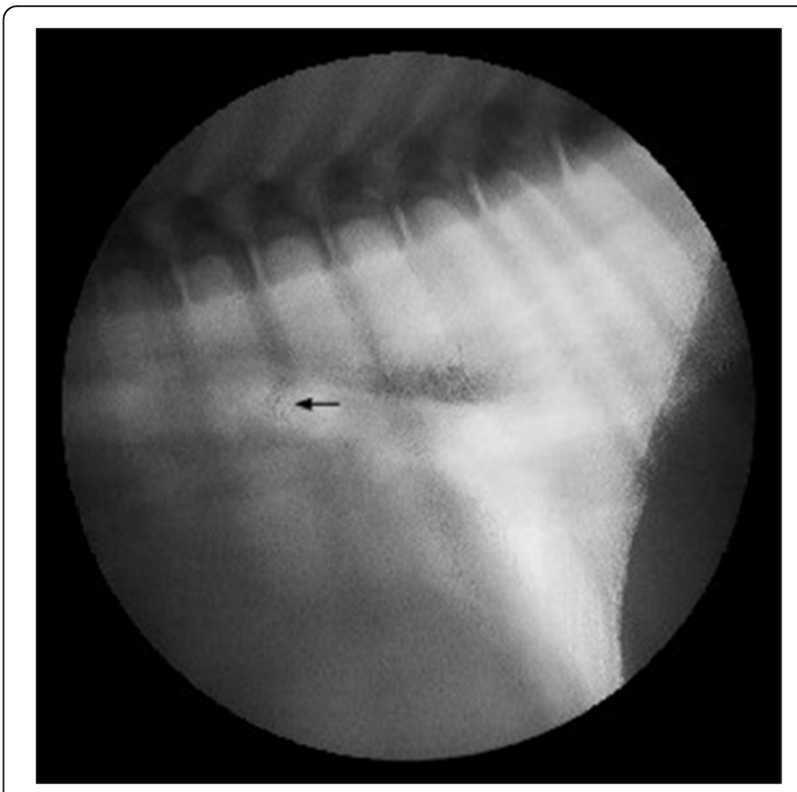

Figure 3 A contrast material swallow study performed under fluoroscopy with the dog in a standing position. In a lateral view of the thorax, a small amount of barium contrast medium diluted $(1: 1)$ with water was seen to escape ventrally (arrow) from the esophagus along a duct-like structure. 
structure and function of the esophagus was demonstrated. The thoracic radiograph obtained after CS demonstrated only a small amount of contrast medium in the lower part of the trachea and in the main bronchus. The finding of the CS study under fluoroscopy was indicative of a TEF. To further evaluate the connection between the trachea and esophagus, a computed tomography (CT) scan was performed. In the non-contrast CT, a 6-mmdeep and 3-mm-wide air-filled pouch was seen in the midline in the bifurcation, at the level of the carina, extending caudally between the main bronchi (Figure 4A and B). The finding was consistent with the hole-like structure seen on bronchoscopy. The esophageal part of the TEF was not visible.

Surgical correction of the TEF was scheduled and amoxicillin-clavulanic acid $^{\mathrm{a}}$ medication was continued. Prior to surgery, a catheter ${ }^{\mathrm{f}}$ was placed along a guide wire ${ }^{\mathrm{g}}$ through the TEF from the trachea into the esophagus under endoscopic control. Ventrodorsal and laterolateral thoracic radiographs subsequently obtained showed that the distal opening of the fistula was located at the level of the sixth intercostal space. The TEF was surgically approached by thoracotomy through the right lateral sixth intercostal space. The esophagus and dorsally lying Nervus vagus were identified. The depth at which the guide wire was running in the esophageal wall and the exact place where the fistula joined the esophagus could not be identified by palpation. The level of the fistular opening was localized by the light of a $9.8 \mathrm{~mm}$ flexible gastroduodenoscope $^{\mathrm{h}}$, which was visible through the esophageal wall. The distal end of the fistula $(0.5 \mathrm{~mm}$ diameter $)$ was bluntly dissected free from the surrounding connective tissue as far cranially as possible (Figure 5). The catheter with guide wire was removed, and the fistula was double ligated with polypropylene 3-0 (Prolene) immediately adjacent to the esophagus. A further double ligature was placed approximately $3 \mathrm{~cm}$ cranially from the first ligatures and the fistula was divided. The fistular tissue was fixed in $10 \%$ non-buffered formalin and submitted for histopathological examination. The dog recovered well from surgery and was discharged the following day. Oral amoxicillinclavulanic acid $^{\mathrm{a}}$ medication was continued for two weeks. Post-operative analgesia was maintained with fentanyl $l^{\mathrm{i}}$ and oral carprofen'.

The histological examination of cross sections of the excised fistular tissue revealed a tube-like structure with a narrow lumen, lined by stratified squamous epithelium. This was underlain by the lamina propria mucosae, a submucosa containing inactive glandular structures, an inner circular and outer longitudinal skeletal muscle layer (muscularis externa) containing (intermyenteric) plexus structures comprised of several unaltered neurons, and the adventitia (Figure 6).

The dog was re-examined two weeks and ten months after surgery, with no evidence of recurring clinical signs. At the first control visit, the blood leucocyte count, arterial $\mathrm{PaO}_{2}$, and CRP were within normal limits, and thoracic radiographs revealed signs of resolving bronchopneumonia. At the second visit, the blood leucocyte count, arterial $\mathrm{PaO}_{2}, \mathrm{CRP}$, and thoracic radiographs showed no features suggesting a disease process. In addition, a CS study using fluoroscopy was performed. This did not detect any evidence of leakage of contrast medium from the esophagus. Both the structure and function of the esophagus appeared unaltered.

\section{Discussion}

A congenital TEF is likely to arise as a result of interruption of the events responsible for elongation and separation of the esophageal and tracheal tubes during the fourth fetal week [7]. In the present report, a congenital malformation is considered most likely due to the dog's young age and the absence of an esophageal foreign body in its history. Histopathology confirms the process to originate from the esophagus and to be patent. The absence of Lamina muscularis mucosae in the fistula

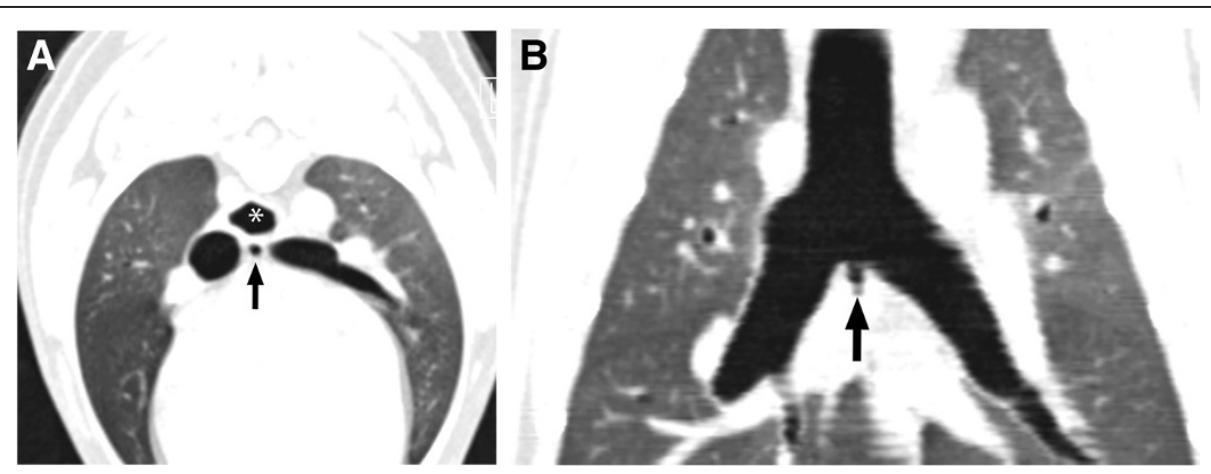

Figure $4 \mathrm{~A}$ thoracic non-contrast CT study of the dog in dorsal recumbency. A) A transverse image at the level immediately caudal to the carina. The TEF (arrow) is visible between the main bronchi. B) A dorsal reconstruction image at the same level as Figure 4A. The proximal part of the TEF (arrow) is seen as a pouch between the main bronchi. 


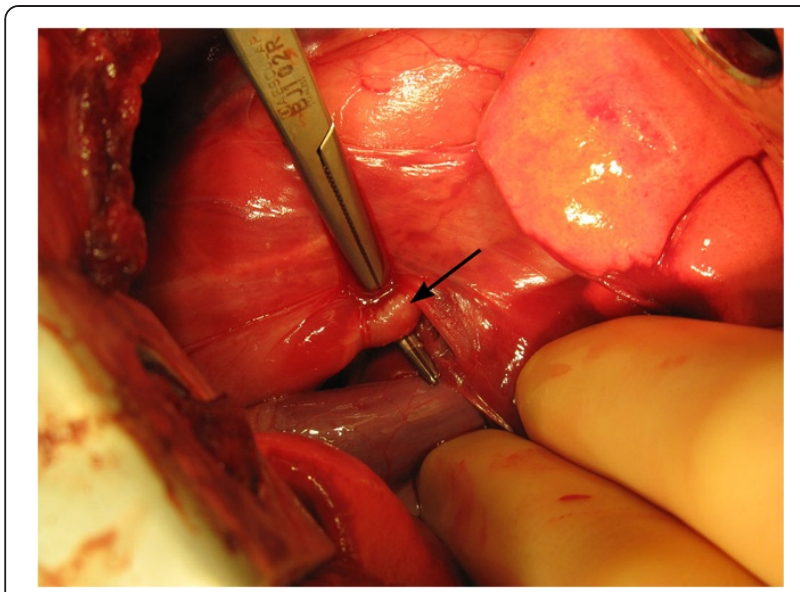

Figure 5 The TEF was approached by thoracotomy through the right lateral sixth intercostal space. The fistula (arrow) was double ligated immediately adjacent to the esophagus and approximately $3 \mathrm{~cm}$ cranially from the first ligatures. The fistula was completely divided and a tissue sample from the fistula was obtained for histopathology.

confirms its origin from the anterior part of the esophagus, since the former is present distally [8]. Furthermore, the lack of any pathological changes in the excised tissue, i.e. absence of a significant inflammatory process and/or abnormal structures, provides strong evidence for its congenital nature $[8,9]$.

This case presented with the classical symptoms encountered in human and canine patients with esophageal fistulas: a chronic cough occurring after drinking due to aspiration of water through the fistula $[3,4,10,11]$, recurrent, chronic lower respiratory tract infections due to continuous aspiration via the fistula [10-13] and gas accumulation in the gastrointestinal tract due to the passage of air from the trachea into the gastrointestinal tract $[2,10,11]$.
The tracheo- and bronchoesophageal fistulas in dogs previously reported were diagnosed with plain thoracic radiographs after barium CS study [2-4,14], indicating that the defect had probably been relatively large in diameter, since a visible quantity of contrast material flowing through it. In the present case, the thoracic radiograph obtained after CS study with diluted contrast medium, demonstrated only a small amount of contrast medium in the lower part of the trachea and in the main bronchus. The presence of contrast material in the airways without visualization of the fistula itself may also be indicative of aspiration and cannot be considered specific for TEF.

In human medicine, numerous diagnostic techniques for TEF have been described, but given the relatively low incidence of TEF; there have been no randomized and controlled trials to support the better use of one method over another. None of the reported diagnostic methods are universally reliable, and the lack of sensitivity makes the diagnosis challenging in mildly affected patients [13,15-19]. The initial radiological investigation consists of plain thoracic and abdominal radiographs, which may raise suspicion of TEF by showing evidence of aspiration and gastric dilatation $[2,12,13]$. The most frequently described radiological diagnostic techniques to confirm the presence of TEF are CS study and the tube esophagogram $[13,15,19]$, where the contrast medium is injected into the esophagus under pressure and observed via plain radiographs and fluoroscopy.

Inconstant patency of TEF is associated in a dog with a slit-like opening of the esophageal fistula orifice [2]. In humans, an esophageal mucosa fold occluding the esophageal side of the fistula, or an oblique course of the fistula to the esophagus has been described, which might cause the esophageal mucosa adjacent to the orifice to function as a valve during swallowing, compressing the anterior esophageal wall against the fistula and occluding its lumen

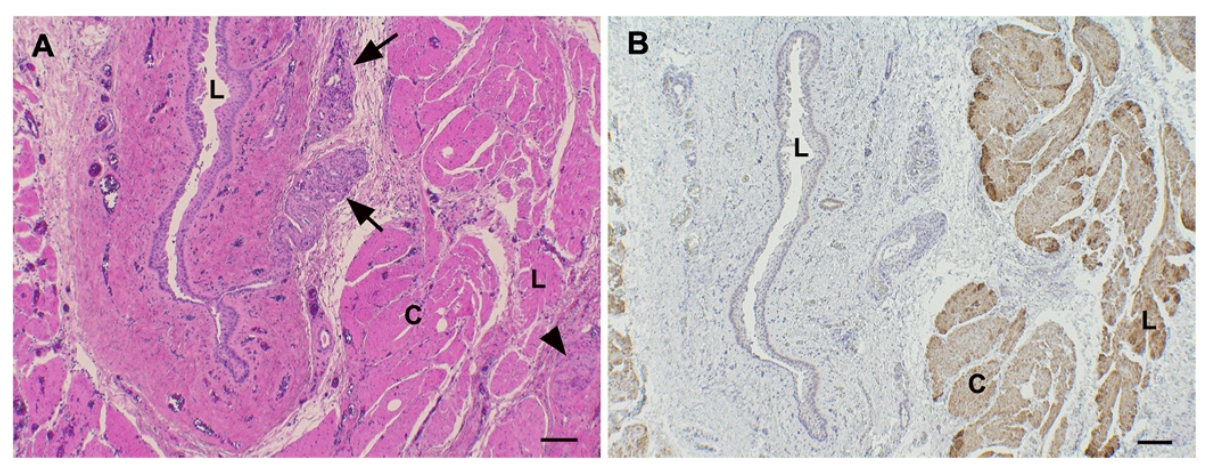

Figure 6 A histological specimen from the fistula. The specimen represents a cross section through the mucosa, submucosa and muscularis externa. The slit-like lumen $(\mathrm{L})$ is lined by squamous epithelium. The submucosa contains inactive glandular structures (arrows), and the muscularis externa is comprised of the inner circular $(C)$ and the outer longitudinal $(L)$ layer, and contains plexus structures comprised of unaltered neurons (arrowhead). A. Hematoxylin-eosin stained section. B. Immunohistology for desmin highlights the striated muscle layers of the muscularis externa and the absence of a lamina muscularis mucosae. Streptavidin peroxidase method, Mayer's hematoxylin counterstain. Scale bars $=100 \mu \mathrm{m}$. $(\times 50)$. 
$[13,20]$. Additionally, a small diameter of the orifice, as in our case, can complicate the diagnosis of TEF. Diluted contrast medium may pass more easily through fistulas with a narrow lumen $[12,14]$, thereby rendering a diagnosis possible in suspected cases where CS study with undiluted contrast material is negative. It should be noted that, as in our case, diluted contrast medium may be more readily observed via fluoroscopy than plain thoracic radiographs.

The use of CT has been increasing in recent years. According to previous studies [12,16,18,21], contrary opinions on the use of $\mathrm{CT}$ in humans exist. A recent study by Mahalik et al. [18] stated that a CT scan could provide good anatomic delineation, but might not help in surgical decision making. To the authors' knowledge, the present case is the first reported study in which CT is used in a dog in an attempt to identify TEF. Noncontrast CT is not helpful in the diagnosis, since it does not enable the visualization of the distal esophageal part of the TEF. However, the air-filled pouch between the main bronchi would raise a suspicion of abnormal anatomy, even if the bronchoscopy does not reveal the orifice of the TEF. In neonate children, the concurrent use of air during CT has been a valuable aid in the diagnosis of TEF, serving as a negative contrast medium [21,22].

Endoscopic techniques, such as bronchoscopy and esophagoscopy, are complimentary techniques for diagnosing TEF. They may allow the fistula to be directly visualized and its origin to be exactly localized $[10,13]$. Nevertheless, endoscopic techniques can lead to a false negative result, and repeated examinations may be necessary, as the fistula can be difficult to identify in a single attempt [13]. In our case, the tracheal wall defect was diagnosed during bronchoscopy. The defect was clearly visible only in the early stage of bronchoscopy, and when later reassessed appeared closed, and as such could easily had been left unrecognized. A reason for poor identification of the fistula could be the small size of the orifice, which might easily collapse due to pressure changes during ventilation. This indicates that a negative diagnosis in endoscopy is not exclusive and other diagnostic methods are needed when TEF is suspected.

In dogs, congenital or acquired bronchoesophageal fistulas are mainly seen in association with an esophageal diverticulum and foreign body [14]. In humans, esophageal atresia is associated with congenital TEF in the vast majority of patients (85-88\%) [23,24]. In our case, the anatomic structure and function of the esophagus were unaltered both prior to and after surgery. This rules out any relevant concomitant anomalies and is considered a good prognostic indicator for surgical correction.

Surgery consists of ligation and division of the fistula and, if needed, repair of the tracheal and esophageal walls $[10,25]$. In the present case, the connection between the trachea and esophagus was identified with a catheter, as previously described $[10,24]$. Thoracotomy was performed in the sixth intercostal space according to guide wire location in thorax radiographs. The proximal end of the fistula was not further searched for due to the moderate length of the fistula, challenging location between important structures, thick connective tissue, and adhesions surrounding the fistula. In humans, surgical repair of the proximally located fistula is approached through the cervical route along the anterior border of the sternocleidomastoid muscle. An approach via thoracotomy is taken when the fistula is located more distally, at the level of the carina, or when partial pulmonary resection is required at the same time $[10,20]$. TEFs of newborns have also been successfully repaired thoracoscopically [26].

Due to the low incidence of reported TEFs in dogs and the lack of long-term follow-up data, no information on complications or the recurrence rate is available. In our case report, the dog's postoperative course was uneventful, with no signs of recurrence within the ten-month followup period. Recurrent TEF is one complication of surgical therapy among children with congenital esophageal atresia and TEF [23]. TEF recurs in less than $5 \%$ to $8 \%$ of cases, most often 2 to 18 months after initial repair [24,25]. Ligation of the fistula rather than complete division increases the incidence of recurrent TEF [25].

\section{Conclusions}

Recurrent respiratory infections in young dogs require a thorough examination to identify possible predisposing pathological conditions. TEF needs to be considered as a possible cause for repeated respiratory tract infections. CS study using fluoroscopy was the most reliable diagnostic method in our case. Diluted contrast material is advisable to use in suspected cases where CS study with undiluted contrast material is negative. Bronchoscopy may allow the fistula to be visualized, but due to a small fistular opening it can lead to a false negative result. When non-contrast CT is not helpful in the TEF diagnosis, air in the esophagus as a negative contrast medium could be more informative. In the case described, surgical correction by ligation and dividing of the fistula remained successful after ten months, and suggests a good prognosis for early diagnosed and operated TEF.

\section{Endnotes}

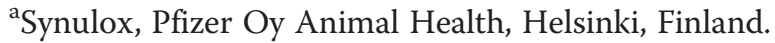

${ }^{\mathrm{b}}$ AmoxClav, Hexal AG, Holzkirchen, Germany.

'Olympus GIF N180, Olympus Medical Systems Europa GMBH, Hamburg, Germany.

${ }^{\mathrm{d}}$ Sodium Chloride 0.9\% Injections USP, B. Braun Medical Oy, Helsinki, Finland.

${ }^{\mathrm{e}}$ Mixobar colon, $1.0 \mathrm{mg} / \mathrm{ml}$, E-Z-EM, Anjou, Canada. 
${ }^{\mathrm{f}}$ Buster Sterile Dog Catheter $1.3 \times 500 \mathrm{~mm}, 4 \mathrm{FG}$, Kruuse, Langeskov, Denmark.

g0.025' guidewire VisiGlide, $2700 \mathrm{~mm}$, Olympus, Hamburg, Germany.

hPentax EG2940K, Pentax GMB, Hamburg, Germany.

${ }^{\mathrm{i}}$ Durogesic, Janssen-Cilag Oy, Espoo, Finland.

${ }^{\mathrm{j}}$ Rimadyl, Pfizer Oy Animal Health, Helsinki, Finland.

\section{Abbreviations}

TEF: Tracheoesophageal fistula; $\mathrm{PaO}_{2}$ : Partial oxygen pressure in the arterial blood; CRP: C-reactive protein; BAL: Bronchoalveolar lavage; CS: Contrast material swallow; CT: Computed tomography.

\section{Competing interests}

None of the authors has any financial or personal relationships that could inappropriately influence or bias the content of this paper.

\section{Authors' contributions}

Clinical examination, diagnosis and treatment of animal were performed by PSK, SJV, MMR and OMLV. AKL performed the analysis and interpretation of the $C T$ findings. AK performed the analysis and interpretation of the histological findings. All the authors helped to draft the manuscript. All authors read and approved the final manuscript.

\section{Author details}

${ }^{1}$ Department of Equine and Small Animal Medicine, Faculty of Veterinary Medicine, Helsinki University, Helsinki, Finland. ${ }^{2}$ Veterinary Pathology, Department of Veterinary Biosciences, Faculty of Veterinary Medicine, Helsinki University, Helsinki, Finland.

Received: 28 April 2013 Accepted: 6 January 2014

Published: 14 January 2014

\section{References}

1. Fingeroth JM: Surgical techniques for esophageal surgery. In Textbook of Small Animal Surgery. 2nd edition. Edited by Slatter. Philadelphia: Saunders; 2003:530.

2. Stogdale L, Steyn DG, Thompson BC: A congenital oesophagotracheal fistula in a two-year-old dog. J S Afr Vet Assoc 1977, 48:212-214.

3. Basher AWP, Hogan PM, Hanna PE, Runyon CL, Shaw DH: Surgical treatment of a congenital bronchoesophageal fistula in a dog. J Am Vet Med Assoc 1991, 199:479-482.

4. Caywood DD, Feeney DA: Acquired esophagobronchial fistula in a dog. J Am Anim Hosp Assoc 1982, 18:590-594.

5. Schebitz $\mathrm{H}$ : X-ray appearance of an esophagotracheal fistula due to foreign body perforation in a dog. Tierarztl Umsch 1960, 15:87.

6. Della Ripa MA, Gaschein F, Gaschein L, Cho DY: Canine bronchoesophageal fistulas: case report and literature review. Compendium 2010, 32:1-10.

7. Ross AJ: Organogenesis of the Gastrointestinal Tract. In Fetal and Neonatal Physiology. 4th edition. Edited by Polin RA, Fox WW, Abman SH. Philadelphia: Saunders; 2011:1188

8. Bacha JW Jr, Bacha LM: Color Atlas of Veterinary Histology. 2nd edition. Baltimore Maryland, Philadelphia: Lippincott Williams \& Wilkins; 2000:119-132.

9. Brown CC, Baker DC, Barker IK: Alimentary system. In Jubb, Kennedy \& Palmer's Pathology of Domestic Animals. 5th edition. Edited by Maxie MG. Edinburg: Elsevier Saunders; 2007:35-36.

10. Genty E, Attal P, Nicollas R, Roger G, Triglia J-M, Garabedian E-N, Bobin S: Congenital tracheoesophageal fistula without esophageal atresia. Int J Pediatr Otorhinolaryngol 1999, 48:231-238.

11. Beasley SW, Myers NA: The diagnosis of congenital tracheoesophageal fistula. J Pediatr Surg 1988, 23:415-417.

12. Hajjar WM, Iftikhar A, Al Nassar SA, Rahal SM: Congenital tracheoesophageal fistula: a rare and late presentation in adult patient. Ann Thorac Med 2012, 7:48-50.

13. Ng J, Antao B, Bartram J, Raghavan A, Shawis R: Diagnostic difficulties in the management of H-type tracheoesophageal fistula. Acta Radiol 2006, 47:801-805.
14. Park RD: Bronchoesophageal fistula in the dog: literature survey, case presentations, and radiographic manifestations. Compend Contin Educ Pract Vet 1984, 6:669-676.

15. Laffan EE, Daneman A, Ein SH, Kerrigan D, Manson DE: Tracheoesophageal fistula without esophageal atresia: are pull-back tube esophagograms needed for diagnosis? Pediatr Radiol 2006, 36:1141-1147.

16. Fitoz S, Atasoy Ç, Yagmurlu A, Akyar S, Erden A, Dindar H: Three-dimensional $\mathrm{CT}$ of congenital esophageal and distal tracheoesophageal fistula in neonates: preliminary results. AJR 2000, 175:1403-1407.

17. Gassner I, Geley TE: Sonographic evaluation of oesophageal atresia and trachea-oesophageal fistula. Pediatr Radiol 2005, 35:159-164.

18. Mahalik SK, Sodhi KS, Narasinhan KL, Rao KLN: Role of preoperative 3D CT reconstruction for evaluation of patients with esophageal atresia and tracheoesophageal fistula. Pediatr Surg Int 2012, 28:961-966.

19. Kirk JME, Dicks-Mireaux C: Difficulties in diagnosis of congenital H-type trachea-oesophageal fistulae. ClinRadiol 1989, 40:150-153.

20. Acosta $J \mathrm{~L}$, Battersby JS: Congenital tracheoesophageal fistula in the adult. Am Thorac Surg 1974, 17:51-57.

21. Wen Y, Peng Y, Zhai RY, Li YZ: Application of MPVR and TL-VR with 64-row MDCT in neonates with congenital EA and distal TEF. World J Gastroenterol 2011, 17:1649-1654.

22. Soye JA, Yarr J, Dick AC, Paterson A: Multidetector-row computed tomography three-dimensional volume reformatted 'transparency' images to define an upper pouch fistula in oesophageal atresia. Pediatr Radiol 2005, 35:624-626.

23. Myers NA, Beasley SW, Auldist AW: Secondary esophageal surgery following repair of esophageal atresia with distal tracheoesophageal fistula. J Pediatr Surg 1990, 25:773-777.

24. Benjamin B: Endoscopy in esophageal atresia and tracheoesophageal fistula. Ann Otol Rhinol Laryngol 1981, 90:376-382.

25. Touloukian RJ: Long-term results following repair of esophageal atresia by end-to-side anastomosis and ligation of the tracheoesophageal fistula. J Pediatr Surg 1981, 16:983-988.

26. Tokhais TA, Zamakhshary M, Aldekhayel S, Mandora H, Sayed S, AlHarbi K, Alqahtani AR: Thoracoscopic repair of tracheoesophageal fistulas: a case control matched study. J Pediatr Surg 2008, 43:805-809.

doi:10.1186/1746-6148-10-16

Cite this article as: Kaminen et al: Management of a congenital tracheoesophageal fistula in a young Spanish water dog. BMC Veterinary Research 2014 10:16.

\section{Submit your next manuscript to BioMed Central and take full advantage of:}

- Convenient online submission

- Thorough peer review

- No space constraints or color figure charges

- Immediate publication on acceptance

- Inclusion in PubMed, CAS, Scopus and Google Scholar

- Research which is freely available for redistribution 